\title{
MEASUREMENT OF THE MECHANICAL STIFFNESS IN CYCLOTORSION OF THE HUMAN EYE
}

\author{
Huibert J. Simonsz, Robert A. Crone, Bob J. de WaAl, \\ Martin Schooneman and Hendrik A. L. Lorentz de HaAs \\ Department of Ophthalmology, Academisch Medisch Centrum and the Netherlands Ophthalmic Research \\ Institute, Amsterdam, Holland
}

(Received 7 Seprember 1983; in revised form 31 January 1984)

\begin{abstract}
We have measured the stiffness in cyclotorsion of the human eye using a scleral suction contact ring mounted on a shalt fitted with an eddy current motor to provide the torque to turn the eye and a shaft-position-encoder to register the torsion. The relation proved to be almost linear within the measuring range in four subjects with constant slope values of about $0.75,0.60,0.55$ and $0.45 \mathrm{~g} \cdot \mathrm{cm} / \mathrm{deg}$. The average, $0.6 \mathrm{~g} \cdot \mathrm{cm} / \mathrm{deg}$, equals $0.5 \mathrm{~g}$ if applied at the radius of the globe. This value is rather low compared to the values obtained by other authors for horizontal eye movements. This may be due to different length-tension characteristics of oblique muscles compared to recti muscles.
\end{abstract}

Eye movements Cyclotorsion Muscle force Orbit Tissue elasticity

\section{INTRODUCTION}

The statics of eye muscles have been investigated in the intact human eye and in detached eye muscles. The relation between length and tension in detached eye muscles was thoroughly examined by Robinson et al. (1969), Scott (1975) and Collins et al. (1969). During strabismus surgery a desinserted lateral rectus muscle was connected to a force transducer and the length-tension relation for that muscle was recorded with the other eye looking into various horizontal directions.

Forces in the intact human eye were assessed more recently by Collins et al. (1981) using a forceps fitted with electronic strain gauges. They found a stiffness of about $1 \mathrm{~g} / \mathrm{deg}$ when the eye was moved with the forceps horizontally from the primary position. This stiffness is the sum of the elasticity of the supporting tissues of the eye and the elasticity of the muscles of the eye. It can be expressed as torque per duction, in units $\mathrm{g} \cdot \mathrm{cm} / \mathrm{deg}$, or $\mathrm{g} / \mathrm{deg}$, assuming that the force is applied at the radius of the globe $(1.2 \mathrm{~cm})$.

We have measured the stiffness in cyclotorsion of the eye using a scleral suction ring mounted on a shaft fitted with an eddy current motor to provide the torque and a shaft-position-encoder to register the torsion.

\section{METHODS}

Four subjects were recruited from colleagues in our department. Three were male and about 30-years old, the fourth subject was female, about 20-years old. The subject was seated in a chair, mounted on a wall of the room. The chair was constructed for photographic measurement of cyclotorsion evoked by body tilt, i.e. the ocular-otolith reflex, so the chair could tilt about an anterior-posterior axis. For these experiments, however, the chair was kept in the upright position. The chair was equipped with a frame extending over and in front of the subject.

On the frame a dental impression holder and a torque-torsion apparatus were mounted, moveable in all directions (Fig. 1). This apparatus was a rebuilt electricity meter (Fig. 2).

This is an eddy current motor consisting of two static coils and a rotating Ferraris disc. The symmetry of the magnetic field of one of the coils is disturbed by applying a voltage across the other coil. This will generate eddy currents in the disc and the disc will rotate. The coil with the variable current was rewound to adapt the apparatus for low-voltage usage. The shaft of the eddy current motor was replaced by a $2 \mathrm{~mm}$ hardened steel shaft with a U-shaped fork brazed onto one end to suspend the contact ring. The shaft was supported by precision micro ball-bearings.

At the other end of the shaft a shaft-positionencoder was mounted (Hohner Elektrotechnik, Tuttlingen, W-Germany), consisting of a transparent disc with 1000 radial, black lines and two fixed light-emitting diodes shining through the edge of the disc onto two light-sensitive transistors. The contact ring was fitted on the fork of the shaft in a sliding fashion. It was turned from teflon, which has a very low coefficient of friction and so will cause the least trouble when an eyelid inadvertently touches the contact ring.

The contact ring consisted of a cylinder with internal and external diameters of 12 and $14.5 \mathrm{~mm}$. The end of the cylinder rested on the sclera near the limbus, but as the horizontal diameter of the cornea is slightly more than $12 \mathrm{~mm}$, the contact ring covered small parts of the cornea, medially and laterally. In 
the wall of the cylinder 40 longitudinal holes were drilled, $0.5 \mathrm{~mm}$ diameter. At the scleral side the holes opened into a groove. The two edges of the groove were $35 \mathrm{deg}$ tilted so as to fit snugly onto the sclera. The other side of the holes opened into a circular canal which was connected by a long flexible tube to a vacuumpump which reduced pressure by maximally 0.5 atmosphere. The sawtoothed pulses from the two channels of the shaft-position-encoder were converted into rectangular pulses and then counted binarily. The two channels were $90 \mathrm{deg}$ out of phase to discern between ex-and incyclotorsion. The digital count ran from 1 to 256 equal to $256 / 1000$ th of a turn, which is about $92 \mathrm{deg}$. Preset buttons were included for calibration and zero setting on numbers 128 and 256 of the binary counter, so the eye position could be registered till about $46 \mathrm{deg}$ in both directions from a zero set position. The digital signal was converted into an analogue signal and fed into a 2-channel $x-t$ recorder (Varian). The a.c. current through the coil with the variable current of the eddy current motor was proportional to the torque generated in the Ferraris disc. The voltage across the coil proved to be proportional to the current, within $5 \%$. The voltage was amplified, converted to d.c. and fed into the other channel of the recorder. The current through the coil of the eddy current motor could be altered manually with two large, wire resistors. The voltage across the second coil was constant, but could be reversed as to reverse the applied torque. The torque of the eddy current motor was calibrated with weights. Informed consent was obtained from all subjects.

The procedure of the measurements was as follows. The eye to be examined was anaesthesized with tetracain $2 \%$. In the first two subjects, a drop of phenylephrine $10 \%$ was used for eyelid retraction. A dental impression was made using a thermoplast on a steel bar which was then fitted onto the frame of the chair. The contact ring was cleansed with ether, wetted and put on the fork. After setting the position of the eye with a fixation-light before the other eye, the contact ring was applied by carefully adjusting the position of the entire torque-torsion apparatus with the pump working. The contact ring was in place when the hissing noise resulting from aspiration of outside air stopped. In the last three measurements the upper eyelid was elevated with a cotton-tipped stick to preclude touch. Torque and torsion were recorded for about $1-2 \mathrm{~min}$ while the applied torque was altered very slowly by turning the variable resistors. After one or two increases one way, the applied torque was reversed. When recording was finished, the pump was switched off and the contact ring was removed. A drop of fluorescein $2 \%$ was applied to check for corneal erosions.

The return of the torsion trace to the starting position was carefully monitored, as incomplete return might indicate slip of the contact ring. Contact between eyelid and contact ring could be detected immediately when the contact ring failed to turn with increasing torque and then suddenly came loose. Measurements in two other subjects failed for this reason.

\section{RESLLTS}

The recordings are depicted in Fig. 3. They show an almost linear increase in torsion with increasing torque, within the recording range. In the first experiment [Fig. 3(a)], the torque was increased in several seconds to about $10 \mathrm{~g} \cdot \mathrm{cm}$ (dashed line). The contact ring was touching the eyelid and there was no smooth increase in torsion. With increasing torque however, the contact ring came loose and at $10 \mathrm{~g} \cdot \mathrm{cm}$ a maximal incyclotorsion was reached of $12 \mathrm{deg}$. When the force was subsequently decreased, the eye did not return to the zero position, because of the contact between eyelid and contact ring. After two increases in torque applied in the incyclo direction, the subject was asked to open the eye wider and no further contact between eyelid and contact ring occurred. The direction of the applied torque was reversed and the eye was rotated three times in the excyclo direction, to a maximum of about $16 \mathrm{deg}$ excyclotorsion. Still the eye did not completely return to the starting position, but lacking only $3 \mathrm{deg}$, most likely due to friction in the ballbearings. The facts that the shape of the peaks is similar and that the final eye position is almost the same as the starting position are indicative of slip being absent. As the contact ring covered small parts of the cornea medially and laterally, movement of the conjunctiva plus contact ring was precluded as well. In the next experiment [Fig. 3(b)] the direction of the applied torque was reversed after each increase. In the first seven increases only one wire resistor was turned with maximal applied torque of about $5 \frac{1}{2} \mathrm{~g} \cdot \mathrm{cm}$. Then twice the torque was increased to $10 \mathrm{~g} \cdot \mathrm{cm}$ both ways. At the end of the experiments the starting position was approached, so no slip occurred. In the last two experiments [Fig. 3(c) and 3(d)] the applied torque was increased only once in both directions to limit the emotional stress of the experiments on the subjects. Maximal torsions were about 15 and 13 deg respectively. In the last case the torsion first rose rapidly, but then reached a plateau when the applied torque was increased further from 7 to $10 \mathrm{~g} \cdot \mathrm{cm}$. Apparently a leash effect was present.

The peculiar shape of the torque traces resulted from the manual operation of the two wire resistors. Because they were first switched on and then turned in sequence, the increase in torque was non-linear. Apart from the two unsuccessful experiments mentioned earlier, no other data were sampled, neither in other subjects nor in the four subjects mentioned above.

The ratio of torque per torsion was about 0.75 , $0.60,0.55$ and $0.45 \mathrm{~g} \cdot \mathrm{cm} / \mathrm{deg}$ in the four subjects. On examination by slitlamp immediately after the experiments, no corneal erosions were seen. The impression 


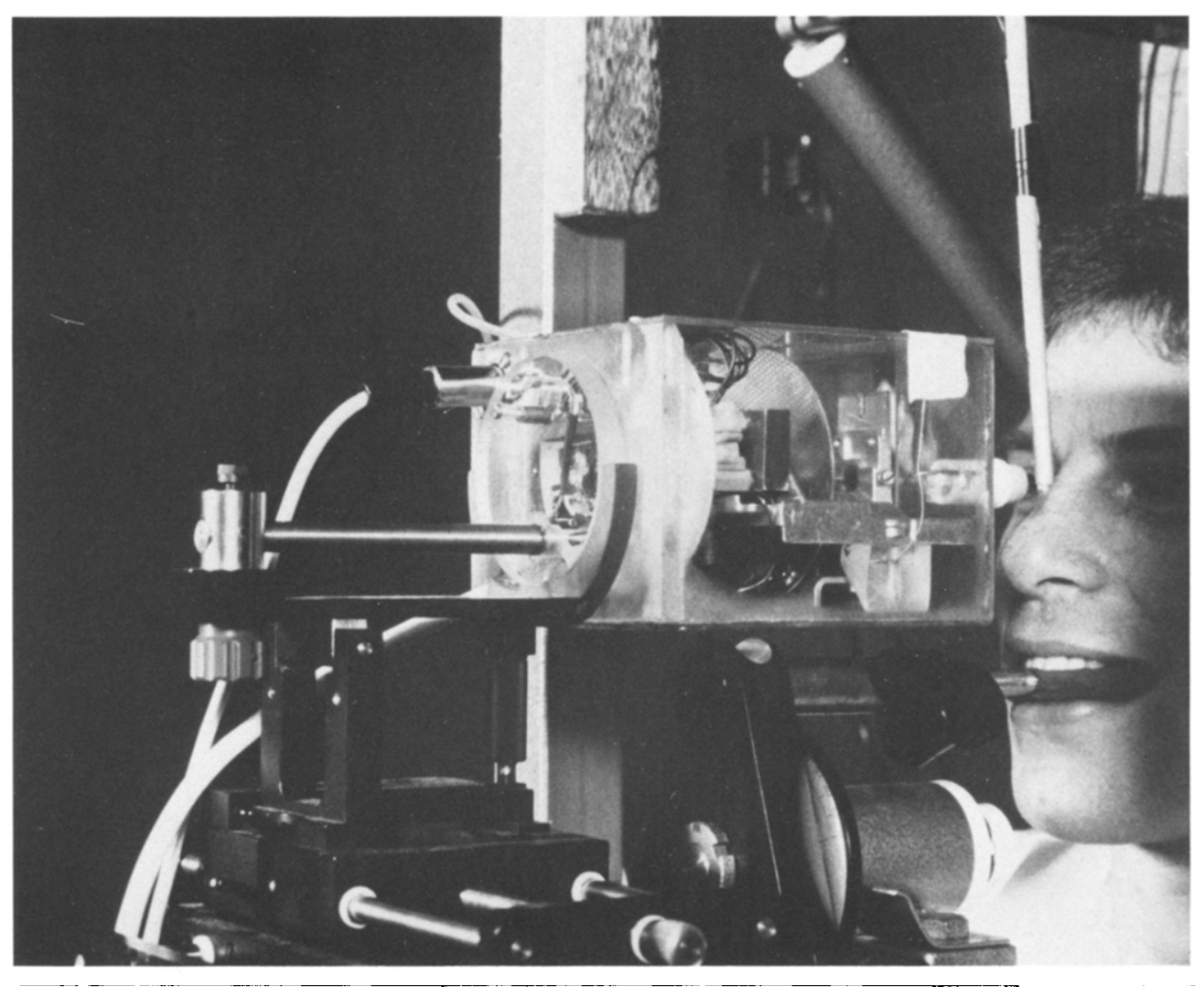

Fig. 1. The test subject bites on a dental impression to fixate the head. The torque-torsion apparatus is moveable in all directions.

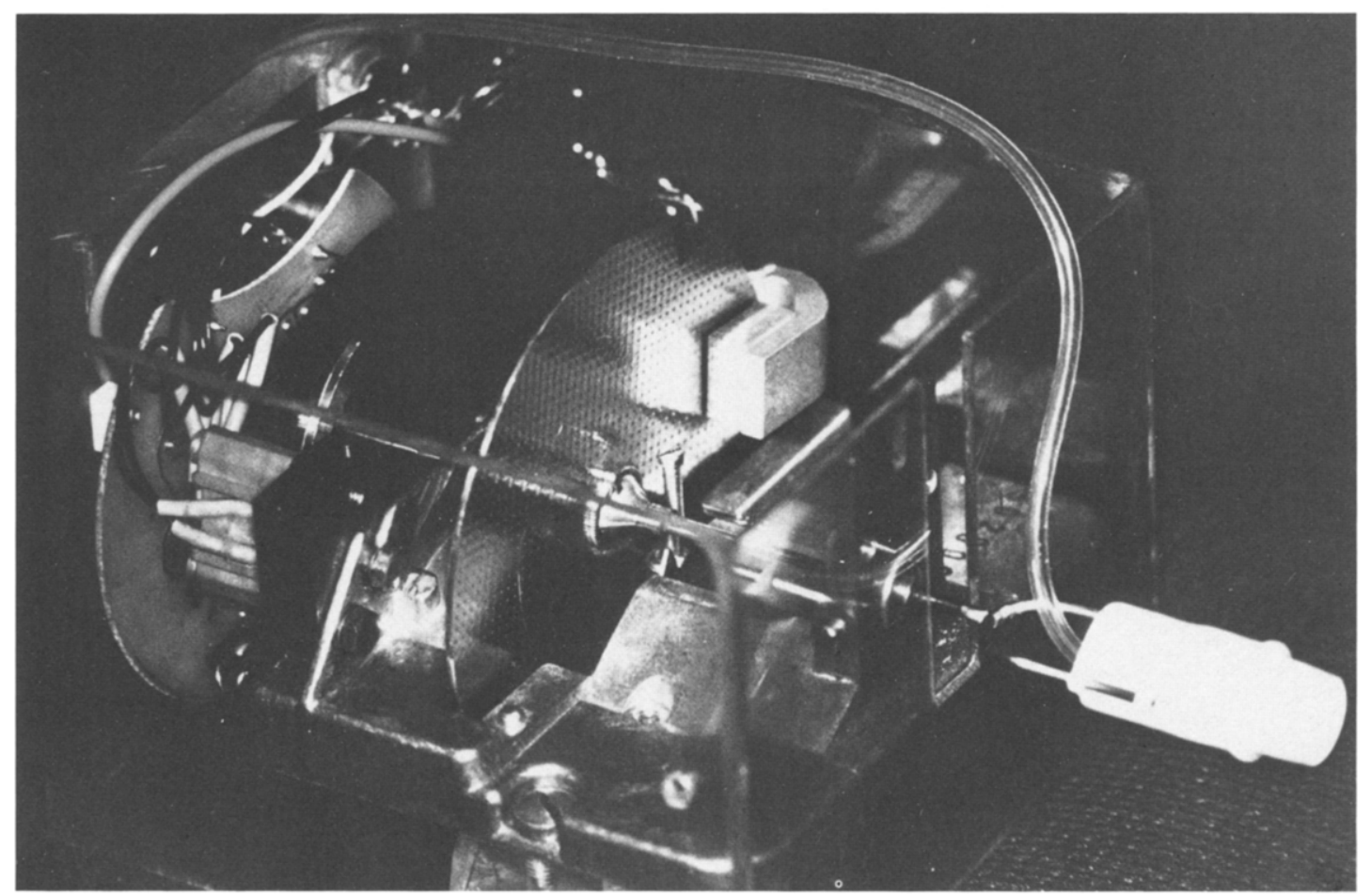

Fig. 2. Close up view of the torque-torsion apparatus. Note teflon contact ring on sliding coupling. Ferraris disc and shaft-position-encoder. 
of the edges of the contact ring was conspicuous however, with minute protrusions of conjunctiva where it had been sucked into the holes. This aspect of the conjunctiva after the experiments hardly permits the view that slip may have occurred either. None of the volunteers experienced any deleterious effects afterwards.

\section{DISCUSSION}

The almost linear relation found between torque and torsion had a constant slope value of about 0.75 ,
$0.60,0.55$ and $0.45 \mathrm{~g} \cdot \mathrm{cm} / \mathrm{deg}$ for the four subjects. The average value of $0.60 \mathrm{~g} \cdot \mathrm{cm}$ deg equals $0.5 \mathrm{~g} / \mathrm{deg}$ when applied at the radius of the globe. or in other words, it takes a force of $0.5 \mathrm{~g}$ to turn the eyc 1 deg, when applied to some point on the equator of the eye. This figure seems rather low compared to the $1.0 \mathrm{~g} / \mathrm{deg}$ Collins et al. (1981) found for moving the intact eye horizontally and the 1.34 (horizontal recti) plus 0.33 (tissue elasticity) $\mathrm{g} / \mathrm{deg}$ found by Robinson et al. (1969). How can we account for this discrepancy? For analysis a Fortran computer model of

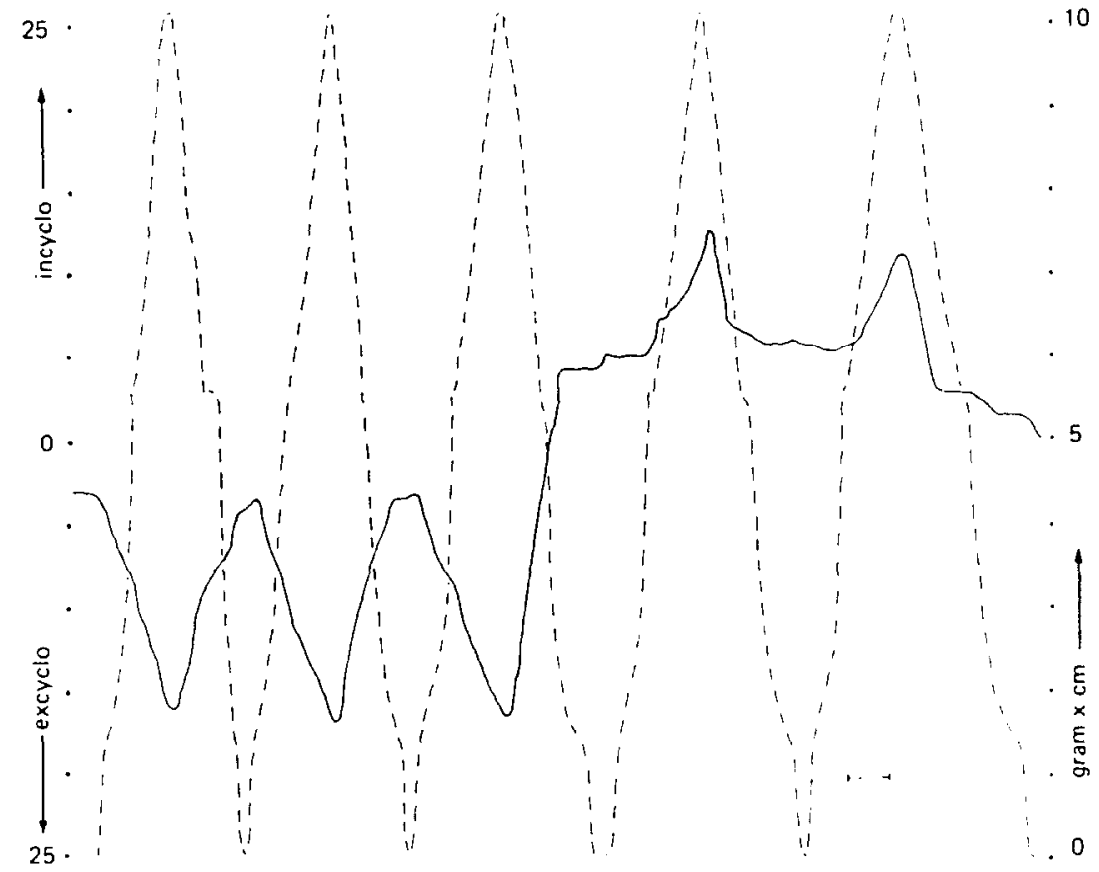

Fig. 3(a).

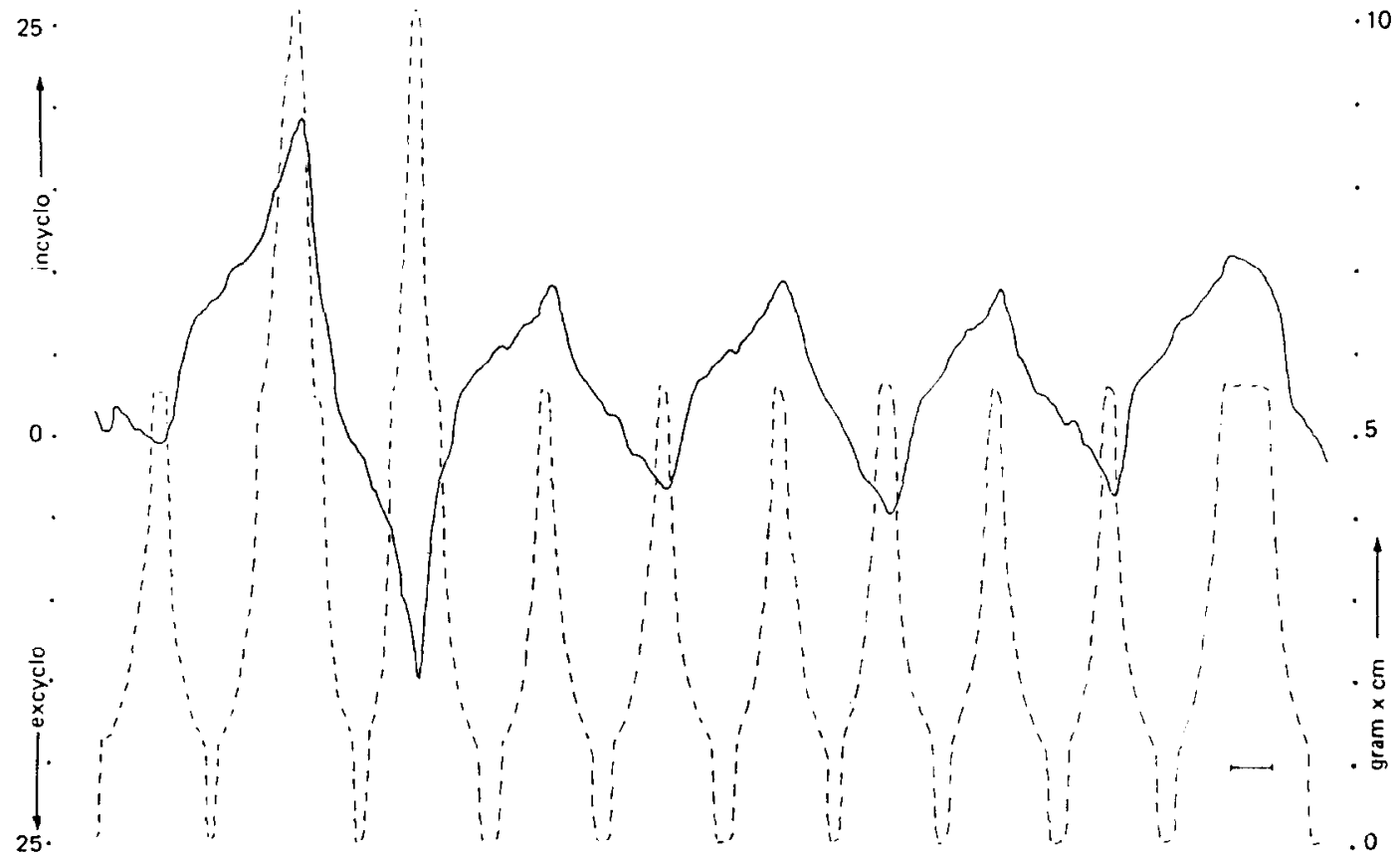

Fig. 3(b). 


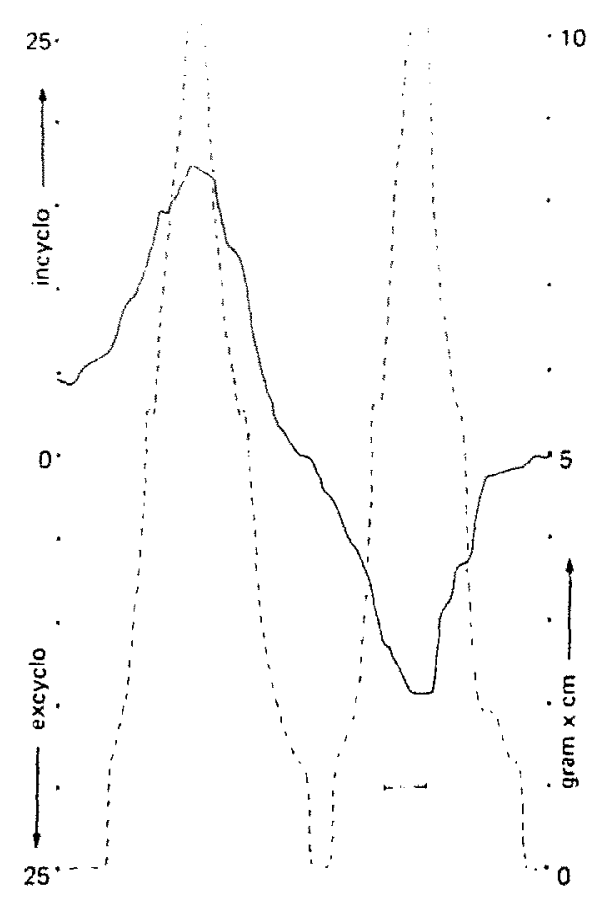

(c)

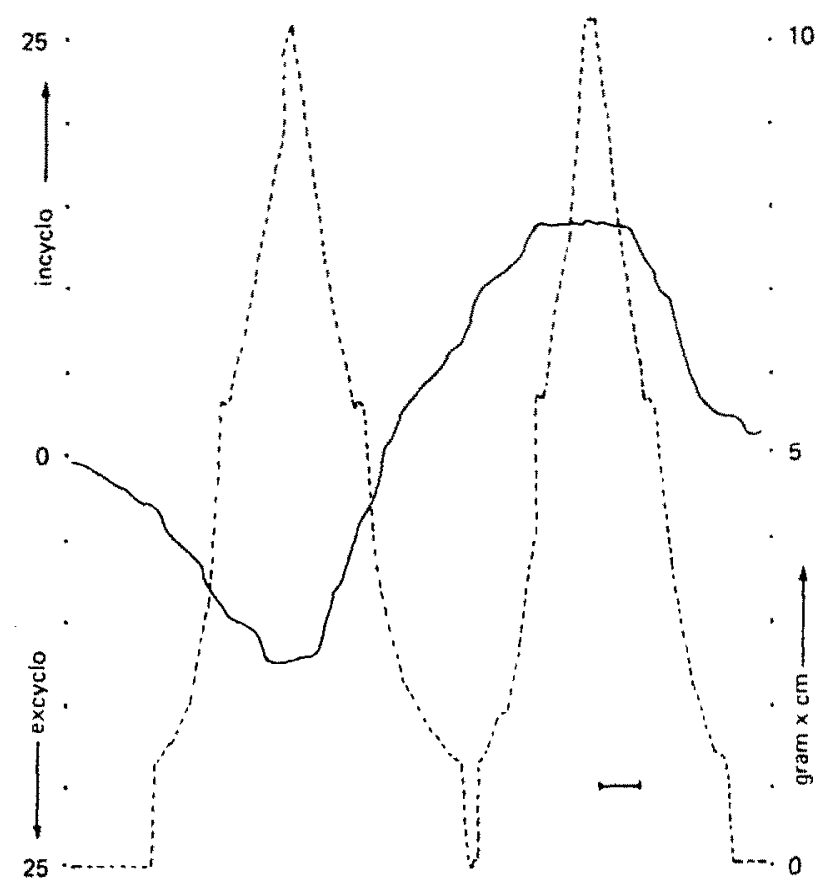

(d)

Fig. 3. Recordings from the four subjects. All left eyes. Read from right to left. Paper-speed: bar equals $4.5 \mathrm{sec}$. Continuous line represents cyclotorsion, dashed line represents applied torque. Full scale cyclotorsion extends from +25 to $-25 \mathrm{deg}$. Full scale torque extends from 0 to $10 \mathrm{~g} \cdot \mathrm{cm}$. The direction of the applied torque is evident from the cyclotorsion reading, but not otherwise indicated.

eye movements was most generously made available to us by Professor D. A. Robinson of the Wilmer Eye Institute in Baltimore. This Fortran program (Robinson, 1975) was entered into the computer of the Netherlands Ophthalmic Research Institute.
The program is based among other things on the measurements by Robinson mentioned earlier (Robinson et al., 1969). The data obtained from measurements on horizontal rocti were extrapolated to vertical recti and obliques by multiplying the 
length-tension characteristics with the relative size of the muscle according to the cross-section area of each muscle.

Surprisingly, if we calculate the torque per cyclotorsion of this model eye, solely on account of the action of the muscles, it amounts to about $1 \mathrm{~g} / \mathrm{deg}$, that is without considering orbital tissue elasticity. This is not in agreement with our results and we must conclude therefore, considering the major role of the obliques in effecting cyclotorsion, that the length-tension characteristics of the obliques are different from those of the recti, even when taking their smaller size into account.

Acknowledgements - We would like to thank Professor D. A. Robinson, Professor G. P. M. Horsten, Dr L. Koornneef, Dr F. Härting, the Department M.V. of G.E.B. Amsterdam (Chief: ir. R. W. Meicr), Miss A. Smits, Miss
S. R. Ompi, D. Bakker. A. Zeilstra and H. Simonsz for their valuable comments, material assistance and volunteering. We would especially like to thank Professor D. A. Robinson. for reading the manuscript.

\section{REFERENCES}

Collins C. C., Scott A. B. and O Meara D. M. (1969) Elements of the peripheral motor apparatus. Am. J. Optom. 46, 510-515.

Collins C. C., Carlson M. R., Scott A. B. and Jampolsky A. (1981) Extraocular muscle forces in normal human subjects. Invest. Ophthal. visual Sci. 20, 652-664.

Robinson D. A., O'Meara D. M. Scott A. B. and Collins C. C. (1969) Mechanical components of human eye movements. J. appl. Physiol. 26, 548-53.

Robinson D. A. (1975) A quantitative analysis of extraocular muscle cooperation and squint. Invest. Ophthal. visual Sci. 14, 801-825.

Scott A. B. (1975) Force and velocity tests in strabismus. Trans. Am. Acad. Ophthal. Otolar. 79, 727-32. 